\title{
Vibrational autoionization of hot bands in methyl iodide
}

\author{
J. Meyer \\ Hamburger Synchrotronstrahlungslabor at Deutsches Elektronen-Synchrotron, Notkestrasse 85, 2000 Hamburg 52, \\ Federal Republic of Germany \\ U. Asaf \\ Racah Institute of Physics, The Hebrew University, Jerusalem 91904, Israel
}

R. Reininger

Synchrotron Radiation Center, University of Wisconsin, 3731 Schneider Drive, Stoughton, Wisconsin 53589

(Received 20 January 1992; revised manuscript received 6 April 1992)

\begin{abstract}
The photoionization of gaseous $\mathrm{CH}_{3} \mathrm{I}$ below the first ionization potential has been studied as a function of pressure and temperature. Structural features that exhibit a Maxwell-Boltzmann temperature dependence are observed as far as $0.17 \mathrm{eV}$ below threshold. They are identified as nd Rydberg transitions originating in excited vibrational levels of the electronic ground state and autoionizing, with $\Delta v=-1$ and -2 , into the vibrational ground state of the ion. From these results along with the simultaneously measured absorption spectrum, we obtained the $v_{2}$ and $v_{3}$ vibrational frequencies of the $d$ Rydberg states.

PACS number(s): 33.20.Ni, 33.80.Eh
\end{abstract}

The photoionization spectra of pure $\mathrm{CH}_{3} \mathrm{I}[1,2]$ and of $\mathrm{CH}_{3} \mathrm{I}$ doped into high-pressure $\mathrm{Xe}[3]$ and $\mathrm{CO}_{2}$ [4] exhibit rich prethreshold structure starting $0.17 \mathrm{eV}$ before the ionization limit. The measured energy spacing between the peaks and the fact that they shifted linearly as a function of the $\mathrm{Xe}$ and $\mathrm{CO}_{2}$ pressure indicated their identity as high Rydberg states and allowed us to determine the electron-scattering lengths in these highly absorbing media $[3,4]$. However, the process leading to their photoionization in the doped systems was not investigated. Ivanov and Vilesov [1] mentioned vibrational autoionization as a possible explanation for the prethreshold signal in pure $\mathrm{CH}_{3} \mathrm{I}$ at pressures below $10^{-3}$ mbar. At higher pressures, they proposed [1] that the photoionization is due to the Hornbeck-Molnar process [5], i.e., $\mathrm{CH}_{3} \mathrm{I}+h v \rightarrow \mathrm{CH}_{3} \mathrm{I}^{*}$ and $\mathrm{CH}_{3} \mathrm{I}^{*}+\mathrm{CH}_{3} \mathrm{I} \rightarrow\left(\mathrm{CH}_{3} \mathrm{I}\right)_{2}^{+}+e$, as observed in the rare gases. Here, $h v$ is the excitation energy, $\mathrm{CH}_{3} \mathrm{I}^{*}$ represents a molecule in a Rydberg state, and $e$ is the emitted electron. It should be noted that in this process, the maxima in the photoionization and in the absorption should coincide, unless strong absorption leads to "dead-layer" effects [6].

In order to identify clearly the nature of the abovementioned transitions in $\mathrm{CH}_{3} \mathrm{I}$ we performed a detailed study of the pressure and temperature dependence in the prethreshold photoionization region. Our results demonstrate that the ionization mechanism is vibrational autoionization with $\Delta v=-1$ and -2 . The former case has been observed in several polyatomic molecules. To our best knowledge, only in $\mathrm{PF}_{3}$ [7] has discrete structure been interpreted as vibrational autoionization with higher changes in vibrational quanta of the ion core.

The experimental setup was described in detail in Ref. [8]. Briefly, monochromatized synchrotron radiation was focused into the center of an experimental cell made of copper or stainless steel. The cells were equipped with entrance and exit $\mathrm{LiF}$ windows and a pair of parallelplate electrodes oriented perpendicular to the windows. This arrangement allowed us to record simultaneously transmission and photoionization spectra in the pressure range $10^{-1}-10^{-5}$ mbar. The spectra were recorded at temperatures $169<T<371 \mathrm{~K}$ with a resolution of 7 $\mathrm{meV}$. The applied electric field was varied between 25 and $250 \mathrm{~V} / \mathrm{cm}$ without observing any effect in the photon energy range of interest.

Figure 1 shows simultaneously recorded absorption

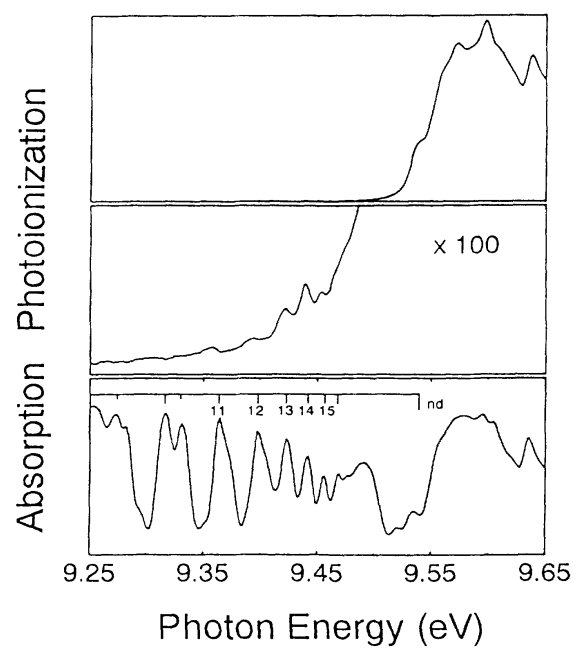

FIG. 1. Absorption (bottom) and photoionization (middle and top) spectra of $0.015-\mathrm{mbar} \mathrm{CH}_{3} \mathrm{I}$ at $300 \mathrm{~K}$. The middle spectrum shows the prethreshold range amplified by a factor of 100. 
and photoionization spectra of $\mathrm{CH}_{3} \mathrm{I}$ in the neighborhood of the first ionization threshold $I_{v}\left({ }^{2} E_{3 / 2}\right)$ at $9.538 \mathrm{eV}$. The amplified photoionization spectrum clearly exhibits five peaks. Their energy positions are listed in Table I together with the energy positions of the nd Rydberg states seen in the absorption spectrum. Based on the energy separation between pairs of adjacent peaks in the photoionization spectrum, one can conclude that they correspond to the Rydberg states $n=11$ to 15 . However, by comparing the peak positions in Fig. 1, or the tabulated values in Table I, one realizes that the peaks in the photoionization spectrum are redshifted by 2 to $3 \mathrm{meV}$ relative to the corresponding peaks in the absorption spectrum. We should emphasize that this result is pressure independent in the whole pressure range investigated. Therefore it can be concluded that the photoionization signal is not due to the Hornbeck-Molnar process.

The effect of temperature in the photoionization signal below threshold is displayed in Fig. 2. As seen in the figure, the overall photoionization signal and the peak heights decrease with a decrease of the sample temperature.

We are now in a position to identify the nature of the peaks in the photoionization spectra. As shown below, they are $n d$ Rydberg transitions originating in excited vibrational levels of the electronic ground state and autoionizing into the vibrational ground state of the ion.

$\mathrm{CH}_{3} \mathrm{I}$ has $C_{3 v}$ symmetry and, consequently, three totally symmetric vibrations $\left(v_{1}, v_{2}, v_{3}\right)$ and three degenerate vibrations $\left(v_{4}, v_{5}, v_{6}\right)$ [9]. Their energies are listed in Table II. Using the energies listed in Table II one obtains that the symmetric vibrational levels of the electronic ground state, ${ }^{1} A_{1}(0,0,1),{ }^{1} A_{1}(0,1,0)$, and ${ }^{1} A_{1}(0,1,1)$, are populated with probabilities $788 \times 10^{-4}, 24.9 \times 10^{-4}$, and $1.9 \times 10^{-4}$ at $300 \mathrm{~K}$. The probability of populating the next higher vibrational level is less than $0.15 \times 10^{-4}$. Some vibrational energies of the ${ }^{2} E_{3 / 2}$ ion have been determined by Ragle et al. [10] and by Karlsson et al. [11] from photoelectron spectra. They are also included in Table II.

A simplified scheme of the vibrational autoionization process is presented in Fig. 3 for the $d$ Rydberg series. We limit the discussion to this series since it is the only one observed in the absorption spectrum in the 9.35-9.55-eV range. Figure 3 shows excitations into $d$ Rydberg states with origin in the vibrational ground state $(0,0,0)$, in the two lowest symmetric vibrational modes $(0,0,1),(0,1,0)$, and in the coupled vibrational mode $(0,1,1)$. As illustrated in the figure (see also Tables I and

TABLE I. Assignment and energy positions (in $\mathrm{eV}$ ) of the peaks observed in the absorption and in the photoionization spectra of $\mathrm{CH}_{3} \mathrm{I}$ shown in Fig. 1 .

\begin{tabular}{lcc}
\hline Level & Absorption & Photoionization \\
\hline $11 d$ & 9.371 & 9.368 \\
$12 d$ & 9.398 & 9.396 \\
$13 d$ & 9.425 & 9.423 \\
$14 d$ & 9.443 & 9.441 \\
$15 d$ & 9.457 & 9.454
\end{tabular}

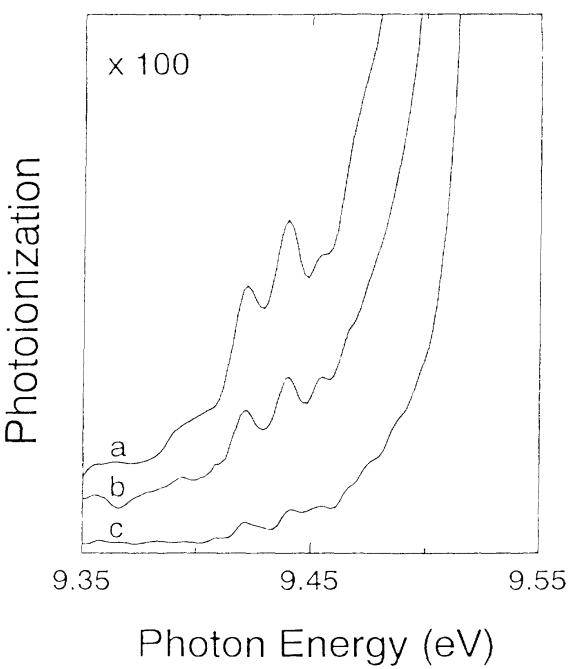

FIG. 2. Photoionization spectra of $0.025-\mathrm{mbar} \mathrm{CH}_{3} \mathrm{I}$ at 371 $\mathrm{K}$ (curve $a$ ), $296 \mathrm{~K}$ (curve $b$ ), and $212 \mathrm{~K}$ (curve $c$ ). The spectra, normalized at $9.597 \mathrm{eV}$, are shown with a one-hundred magnification and staggered vertically.

II), the total energy of the system after excitation from the ${ }^{1} A_{1}(0,1,0)$ level into Rydberg states beginning at the $12 d(0,1,0)$ is larger than that of the ${ }^{2} E_{3 / 2}$ vibrational ground state of the ion. Therefore autoionization fulfilling the propensity rule $\Delta v=-1$ is possible for all these transitions by the conversion of one quantum vibrational energy into electronic energy as discussed by Berry in the case of $\mathrm{H}_{2}$ [12]. As seen in Fig. 3 (see also Tables I and II), vibrational autoionization is also possible for excitations from the ${ }^{1} A_{1}(0,1,1)$ level into Rydberg states beginning at the $11 d(0,1,1)$. In this case, the two vibrational quanta of the couple mode are transferred to the Rydberg electron. The amplified photoionization spectrum shown in Fig. 1 reveals that these are, indeed, our experimental results. The first peak in the spectrum corresponds to the $11 d(0,1,1)$ autoionizing Rydberg state, the subsequent ones have contributions from the vibrational autoionization of the $n d(0,1,1)$ and $(n+1) d(0,1,0)$ Rydberg states originating in the ${ }^{1} A_{1}(0,1,1)$ and ${ }^{1} A_{1}(0,1,0)$ levels, respectively. We note in passing that the energy of the first vibrational autoionizing Rydberg state with origin in the ${ }^{1} A_{1}(1,0,0)$ level is $9.472 \mathrm{eV}$. As seen in Figs. 1 and 2, the strongly increasing background prevents us from resolving Rydberg states with excitation energies greater than $9.47 \mathrm{eV}$ and, in particular, those with origin in the ${ }^{1} A_{1}(1,0,0)$ level.

The shift in energy positions between the absorption

TABLE II. Vibrational frequencies of the ground $\left({ }^{1} A_{1}\right)$ and ionic $\left({ }^{2} E_{3 / 2}\right)$ states (in meV).

\begin{tabular}{lccccccr}
\hline \hline State & $v_{1}$ & $v_{2}$ & $v_{3}$ & $v_{4}$ & $v_{5}$ & $v_{6}$ & Ref. \\
\hline${ }^{1} A_{1}$ & 366 & 155 & 66 & 379 & 178 & 109 & {$[9]$} \\
${ }^{2} E_{3 / 2}$ & & 157 & 61 & 379 & & 114 & {$[11]$} \\
${ }^{2} E_{3 / 2}$ & 376 & 152 & & & & & {$[10]$} \\
\hline
\end{tabular}




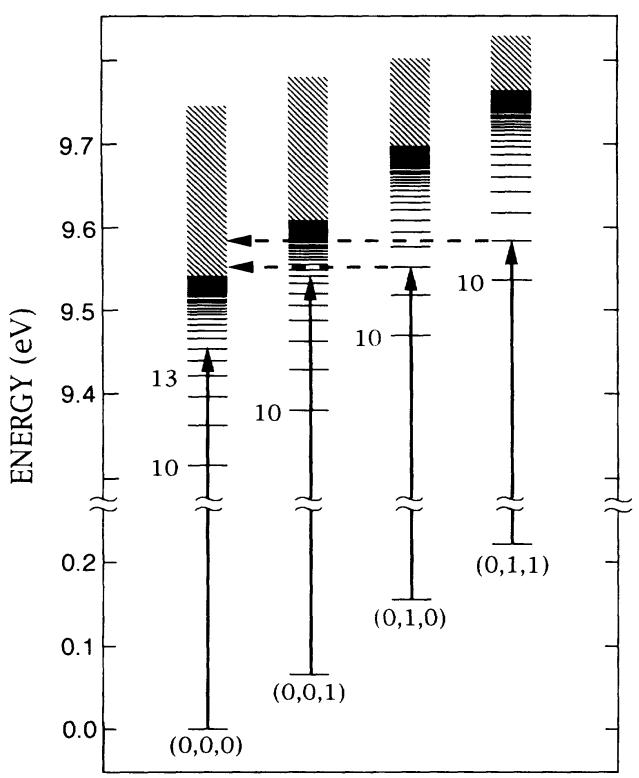

FIG. 3. Energy-level diagram showing transitions originating in the vibrational levels of the electronic ground state and terminating in $n d$ Rydberg levels. The dashed horizontal arrows indicate the experimentally observed onset for autoionization into the continuum of the ionic ${ }^{2} E_{3 / 2}$ vibrational ground state.

and the photoionization maxima seen in Fig. 1, and summarized in Table $I$, is now evident. It is due to the difference between the vibrational energies of the Rydberg states and those of the ${ }^{1} A_{1}$ electronic ground state. From the energy shifts we obtain $153 \mathrm{meV}$ for the $v_{2}^{\prime}$ vibrational mode and, based on this value, $65 \mathrm{meV}$ for the $v_{3}^{\prime}$ vibrational mode. The former value is in between those determined by Ragle et al. [10] and by Karlsson et al. [11] for the $v_{2}^{+}$vibrational mode of the ${ }^{2} E_{3 / 2}$ ion. The $v_{3}^{\prime}$ mode is slightly lower than the value obtained for the corresponding mode of the ion [11].

Additional evidence proving that the peaks in the prethreshold photoionization are vibrational autoionizing Rydberg transitions originating in excited vibrational levels of the electronic ground state and autoionizing into the vibrational ground state of the ion is obtained from the evaluation of the results presented in Fig. 2. The ratios of the integrated areas below the peaks with $n \geq 12$ at 371,296 , and $212 \mathrm{~K}$ are 3:1:0.2. Clearly, the main contribution to these peaks arises from Rydberg states which originate in the ${ }^{1} A_{1}(0,1,0)$ level. The calculated ratios for the population of the ${ }^{1} A_{1}(0,1,0)$ level at 371,296 , and $212 \mathrm{~K}$ are $3.4: 1: 0.1$, in good agreement with the experimental ratios.

As seen in Figs. 1 and 2 the autoionizing Rydberg states are superimposed on a background which increases with photon energy and with temperature. Several processes are responsible for this background. Among these are the direct ionization from the vibrational levels of the ${ }^{1} A_{1}$ state into the vibrational ground state of the ${ }^{2} E_{3 / 2}$ ion. For the vibrational levels $(0,0,1),(0,1,0)$, and $(0,1,1)$ these onsets are at $9.472,9.383$, and $9.317 \mathrm{eV}$, respectively. As seen in Figs. 1 and 2, it is possible to identify steps in the photoionization spectra at these energies. Another contribution to the background is, most probably, rotational autoionization [13]. However, in order to explain photoionization at $\approx 9.47 \mathrm{eV}$ by this process, one has to assume a transfer of more than 10 units of angular momentum to the Rydberg electron. Such a high transfer of angular momentum should be feasible by a multistep process which is possible in the long-time scale of our measurements.

In summary, based on our experimental results we were able to demonstrate that most of the structure in the photoionization spectrum of $\mathrm{CH}_{3} \mathrm{I}$ below threshold is due to vibrational autoionization of hot bands. From the energy shifts observed between the absorption and photoionization spectra we determined the energy of the $v_{2}^{\prime}$ and $v_{3}^{\prime}$ vibrational modes of the $n d$ Rydberg series.
[1] V. S. Ivanov and F. I. Vilesov, Opt. Spektrosk. 39, 857 (1975) [Opt. Spectrosc. (USSR) 39, 487 (1975)].

[2] A. Köhler, Ph.D. thesis, Hamburg University, 1987 (unpublished).

[3] I. T. Steinberger, U. Asaf, G. Ascarelli, R. Reininger, G. Reisfeld, and M. Reshotko, Phys. Rev. A 42, 3135 (1990).

[4] U. Asaf, I. T. Steinberger, J. Meyer, and R. Reininger, J. Chem. Phys. 95, 4070 (1991).

[5] J. A. Hornbeck and J. P. Molnar, Phys. Rev. 84, 621 (1951).

[6] R. Reininger, V. Saile, and P. Laporte, Phys. Rev. Lett. 54, 1146 (1985).

[7] J. Berkowitz and J. P. Greene, J. Chem. Phys. 81, 4328
(1984).

[8] J. Meyer, R. Reininger, and U. Asaf, Chem. Phys. Lett. 173, 384 (1990).

[9] G. Herzberg, Infrared and Raman Spectra of Polyatomic Molecules (Van Nostrand, New York, 1945).

[10] J. L. Ragle, I. A. Stenhouse, D. C. Frost, and C. A. McDowell, J. Chem. Phys. 53, 178 (1970).

[11] L. Karlsson, R. Jadrny, L. Mattsson, F. T. Chau, and K. Siegbahn, Phys. Scr. 16, 225 (1977).

[12] R. S. Berry and S. E. Nielsen, Phys. Rev. A 1, 395 (1970).

[13] B. Ruscic and J. Berkowitz, J. Chem. Phys. 93, 1747 (1990). 